\title{
Les pratiques déclarées des enseignants sur leur enseignement grammatical : quelles élaborations pour quelles transmissions des discours métalinguistiques?
}

Marie-Eve Damar

\section{(2) OpenEdition Journals}

Édition électronique

URL : http://journals.openedition.org/rdlc/2683

DOI : $10.4000 /$ rdlc. 2683

ISSN : 1958-5772

Éditeur

ACEDLE

Référence électronique

Marie-Eve Damar, «Les pratiques déclarées des enseignants sur leur enseignement grammatical : quelles élaborations pour quelles transmissions des discours métalinguistiques? », Recherches en didactique des langues et des cultures [En ligne], 15-1 | 2018, mis en ligne le 02 janvier 2018, consulté le 01 mai 2019. URL : http://journals.openedition.org/rdlc/2683 ; DOI : 10.4000/rdlc.2683

Ce document a été généré automatiquement le 1 mai 2019.

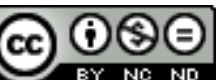

Recherches en didactique des langues et des cultures is licensed under a Creative Commons AttributionNonCommercial-NoDerivatives 4.0 International License 


\title{
Les pratiques déclarées des
} enseignants sur leur enseignement grammatical : quelles élaborations pour quelles transmissions des discours métalinguistiques?

\author{
Marie-Eve Damar
}

\section{Introduction}

1 Le vieux « silence métalinguistique » (Ladmiral, 1975) ne semblant plus de mise dans les classes de FLE (Français Langue Etrangère), l'enseignement grammatical explicite peut s'opérer de plusieurs manières : déductive, inductive, par la conceptualisation. Elle est traditionnellement déductive, ce qui signifie, de manière très synthétique : l'enseignant fournit la règle. Pour pallier une certaine passivité des apprenants durant les activités de grammaire explicite, certains lui préfèrent une démarche de découverte, qui peut être considérée comme la traduction didactique de l'adage « on retient mieux ce que l'on a contribué à découvrir », et répond à une injonction pédagogique plus moderne : mettre l'apprenant au centre de ses apprentissages.

2 Après un éclairage théorique sur la problématique et les enjeux du discours grammatical explicite, cette contribution entend, par le biais d'une enquête auprès d'enseignants de FLE belges francophones, apporter un éclairage sur les pratiques déclarées des enseignants concernant ces démarches d'enseignement de la grammaire. La démarche déductive est-elle plus répandue? $\mathrm{Ou}$, au contraire, les enseignants s'essaient-ils à l'induction, à la conceptualisation ? Par le biais de quelles activités? 


\section{La démarche déductive (ou expositive)}

3 Les méthodologies traditionnelles, comme la méthode grammaire-traduction, proposent une approche déductive, ou expositive. On nous pardonnera ce raccourci, mais exposer cette méthodologie de façon détaillée (voir notamment Germain, 1993) nous éloignerait de notre objectif: elle correspond prototypiquement à une séquence pédagogique où l'enseignant expose la règle, qui est ensuite appliquée dans des exercices par les apprenants.

4 Si la créativité en est absente et la conception de l'enseignement très scolaire, on a également reproché à cette approche de bloquer la communication en focalisant l'attention sur la forme. Les activités liées à ce type de démarche sont souvent perçues comme laissant peu de place à la créativité, puisqu'elle supposent une « remise en ordre psychologique », à l'opposé du « désordre » des activités de communication, de groupe, de jeux de rôle (Flament-Boistrancourt, 1994). Force est de constater néanmoins qu'elles occupent jusqu'à ce jour une place non négligeable dans les manuels de FLE, par exemple, pour n'en citer que deux, dans Alter Ego 1 et Version Originale A2.

5 Si les recherches en acquisition des langues participent à l'élaboration d'une didactique interventionniste, on peut interroger la discipline sur l'éventuelle supériorité d'une approche inductive de la grammaire sur une approche déductive. Les études en psycholinguistique tendent à montrer que les deux approches se valent, ou même que, contrairement à ce qu'on pourrait penser au vu de la volonté actuelle de faire participer l'apprenant à l'élaboration du savoir, l'approche déductive serait supérieure (Seliger, 1975 ; De Keyser, 1995). Ces résultats sont à nuancer, et on sait combien les recherches sur l'évaluation de l'efficacité des méthodologies restent difficiles à mettre en place.

6 Mais qu'en est-il des pratiques de classe? Les enseignants déclarent-ils mobiliser généralement la démarche déductive, comme l'étude de Fougerouse le montrait en 2001, ou préfèrent-ils une approche inductive?

\section{La démarche inductive}

7 À cette passivité des apprenants durant les activités de grammaire explicite a succédé une démarche de découverte, inductive. Celle-ci s'apparente à une sorte de maïeutique pour faire deviner la règle détenue jusqu'alors par l'enseignant, à partir d'un corpus d'exemples choisis pour mettre en avant la régularité linguistique visée par l'apprentissage. Cette démarche suppose également d'emblée un apprentissage guidé: pas de démarche inductive sans accompagnement de l'apprenant dans la formulation de ses hypothèses, ce qui exclut les apprentissages et les outils autodidactiques.

8 La démarche d'induction peut être rapprochée de ce que Sharwood Smith (1981) et Ellis (1993) nomment les activités de conscientisation relatives à la grammaire (consciousnessraising), qui concernent les activités de classe relatives à la réflexion et la compréhension du code. Avec l'aide de l'enseignant, l'apprenant tente de découvrir le fonctionnement linguistique et construit sa propre grammaire explicitée. Signalons que les apprenants cherchent à reconstruire la règle grammaticale détenue par l'enseignant, et la sélection et l'organisation des données orientent l'induction. 
Une démarche inductive, qui semble avoir la préférence des didacticiens comme, nous le verrons, des enseignants (ceux-là influençant peut-être ceux-ci), se traduit par la présentation d'un corpus à examiner. Ces données linguistiques sont sélectionnées soigneusement, et tendent à illustrer la règle à faire deviner par les apprenants. Par exemple, on montre pour expliciter la différence entre le futur simple et le futur périphrastique, des exemples où le premier renvoie à un procès éloigné temporellement dans le futur et où le second réfère à une temporalité plus proche (Ce soir, nous allons danser versus Dans dix ans, nous ferons le tour du monde). L'apprenant infère de cette façon le fonctionnement linguistique - supposé - du futur simple et du futur proche, qui correspond à la terminologie: le futur proche est plus proche temporellement que le futur simple.

10 Ce discours, qui convoque ici, relevons-le en passant, une terminologie faisant entendre le sens retenu pour la forme, n'est pas linguistiquement recevable. Les contre-exemples abondent (Après le travail, j'irai faire des courses versus Dans trente ans, je vais prendre ma retraite). On peut illustrer également cette invalidité des discours proposés avec d'autres faits grammaticaux, comme celui des articles définis et indéfinis (Damar, Roig, Van Raemdonck, 2010a), où le choix des exemples dans les manuels et les grammaires illustre l'opposition connu/non connu, qui ne correspond pas cependant au sens des formes (on explique Je vois UN arbre par LA fenêtre sinon par une différence de vision: partitive vs exhaustive), ou encore avec l'exemple du subjonctif (Damar, 2008).

11 Toute règle induite reflète une description linguistique, toujours conditionnée par les représentations linguistiques des enseignants et les ouvrages de référence qu'ils utilisent : il ne faudrait pas croire qu'une démarche inductive donnerait un accès plus direct à la langue qu'une démarche déductive.

Les représentations des enseignants sur le système linguistique du français engendrent des répercussions sur l'enseignement. Par exemple, avec la langue espagnole, le genre grammatical peut recevoir un traitement sexué, comme le souligne Kasazian (2017). On constate cette fois encore une confusion dans le discours de l'enseignant entre la langue et la réalité. Ce genre grammatical sexué constitue une sorte de stéréotype linguistique qui est socialement partagé mais linguistiquement erroné. Quelles répercussions cette vision faussée de la langue peut-elle entrainer pour les apprenants?

L'amalgame entre genre biologique et genre grammatical n'est donc pas anodin et peut gêner l'apprenant, jusque dans l'exercice et l'assimilation des flexions verbales dans le cas de l'apprentissage de l'espagnol (Kasazian, $2017: 280$ ).

Cet imaginaire linguistique (pour reprendre l'expression de Houdebine) se définit comme :

le rapport du sujet à la langue, la sienne et celle de la communauté qui l'intègre comme sujet parlant-sujet social ou dans laquelle il désire être intégré, par laquelle il désire être identifié par et dans sa parole ; rapport énonçable en termes d'images, participant des représentations sociales et subjectives, autrement dit d'une part des idéologies (versant social) et d'autre part des imaginaires (versant plus subjectif). (Houdebine, 2002: 10)

Pour éclairer ce phénomène, on pourra convoquer le concept de représentation sociale et plus largement le cadre théorique de la psychologie sociale, qui est lié aux travaux de Moscovici (notamment 1984), le père fondateur de la théorie des représentations sociales (TRS). Ce concept désigne : 
une forme de connaissance spécifique, le savoir de sens commun, dont les contenus manifestent l'opération de processus génératifs et fonctionnels socialement marqués. (Jodelet, 1984 : 367)

15 Cette dimension des représentations sociales mériterait des recherches approfondies. Constate-t-on une convergence de ces différentes représentations? Pour quels faits de langue? Quelle est la validité linguistique de ces représentations? Étant donné que bon nombre d'activités grammaticales les révèle, voire les conditionne, il est crucial de s'y attacher, et cela indépendamment du type de démarche grammaticale adopté.

La démarche inductive avoisine également la conceptualisation.

\section{La conceptualisation}

17 Il s'agit d'un autre type d'activités inductives, proposées par Besse dès la fin des années 1960. Celui-ci nomme ces activités de conceptualisation «exercices », mais nous gardons au mot « exercice » l'idée de "s'exercer », donc de s'entraîner de manière répétitive en vue de l'acquisition d'une compétence. Notre définition nous semble donc incompatible avec l'activité de conceptualisation telle que la conçoit Besse. La démarche qu'il propose s'apparente plutôt à une réflexion sur le fonctionnement langagier. Ensuite, comme le signalent Besse et Porquier, on ne peut la qualifier d'exercice vu qu'elle n'est pas reproductible à l'identique (Besse \& Porquier, 1991 : 115).

Il s'agit ici de partir d'erreurs commises par les apprenants, reflets de leur interlangue, pour qu'ils élucident les règles d'un microsystème. Les apprenants, s'appuyant sur leurs intuitions et leur grammaire intériorisée, échafaudent des hypothèses sur le fonctionnement de la langue. Les savoirs concernant la langue ont déjà été intériorisés par l'apprenant, comme l'explique Berthoud, qui décrit les activités de conceptualisation :

L'explication métalinguistique ne vise donc pas ici à enseigner (déductivement) des règles de la langue, mais cherche à consolider des savoirs déjà partiellement intériorisés par une réflexion menée par les apprenants, en fonction des moyens dont ils disposent déjà et selon leurs propres besoins d'apprentissage. (Berthoud, $1993: 75)$

\section{Induction et conceptualisation}

Les différences avec les activités liées à la démarche inductive sont que d'une part la conceptualisation des apprenants concerne leurs propres règles, là où l'apprenant dans la démarche inductive cherche à trouver la règle telle que le professeur ou le manuel l'a conçue ; et d'autre part l'élaboration du contenu de la démarche de conceptualisation est liée à l'interlangue de l'apprenant.

"Tentative isolée, mais intéressante » juge Vigner dans les années quatre-vingt (1984 : 89). Cette approche n'est pas beaucoup exploitée en classe de langue, comme le constate Vigner quelque vingt années plus tard (2004:126). Cette démarche maïeutique comporte beaucoup d'exigences. Elle demande au professeur une formation linguistique solide, une grande souplesse sur la progression des contenus d'enseignement et du côté des apprenants, un bagage scolaire de grammaire explicitée et un intérêt pour la réflexion métalinguistique.

Or, les apprenants de FLE ne se montrent pas toujours capables d'extraire d'un corpus une règle grammaticale : «(...) les apprenants qui n'ont pas eu de formation grammaticale durant leur scolarité seront moins aptes (et moins intéressés) à mener des exercices de conceptualisation » [...]. (Besse \& Porquier, 1991 : 114) 
20 portera en lui les traces des représentations grammaticales de cet enseignant. C'est pourquoi, finalement, il nous semble que l'on se rapproche de la démarche inductive " classique », qui ne dévoilerait pas ses cartes.

21 Ces deux démarches, induction et conceptualisation, impliquent pour les apprenants un intérêt pour la réflexion grammaticale, une motivation à la découverte des règles et à leur formalisation. Force est de constater que cette pratique pédagogique est loin d'être applicable à tous les publics. La démarche de conceptualisation est proprement didactique, et prend du temps à être mise en place, puisqu'elle nécessite différentes phases: examen et analyse des occurrences, déduction des régularités et du sens grammatical, mise en commun et négociation d'un métadiscours.

Si l'objectif poursuivi par l'enseignement est l'acquisition d'une compétence de communication, on peut douter du fait que la réflexion et la négociation grammaticales soient nécessaires: si l'on peut apprendre à parler une langue sans avoir appris sa grammaire, celle-ci est nécessaire à l'orthographe, mais on peut apprendre les règles sans y réfléchir pour autant. Et si on opte pour ce type d'enseignement, la plus-value acquisitionnelle est-elle suffisante pour que l'on prenne le risque d'empiéter sur d'autres activités, plus communicatives? Cuq (1996 : 87-101). L'exploitation par le professeur des manuels présentant des exercices de conceptualisation pourrait toutefois renvoyer à la grammaire inductive et non à la démarche de conceptualisation, à partir des mêmes matériaux didactiques, nous avons déjà fait état de cette réalité :

$\mathrm{Au}$ final, les activités de conceptualisation relèvent de la pratique de classe, et dépendent de l'enseignant et de sa conception de l'enseignement : le concepteur du manuel ne contrôle pas les exploitations de son ouvrage. (Damar, 2008 : 67)

Par ailleurs et plus généralement, ces méthodologies, qu'elles soient déductives, inductives, ou de conceptualisation, engendrent cependant toujours un clivage gênant entre les activités de classe : ludiques versus contraignantes. L'articulation dans les leçons des activités grammaticales et de communication demeure une problématique récurrente depuis le retour de la grammaire (Coste, 1985) après les approches visant à l'éviter. Les méthodes d'enseignement actuelles, preuves en sont les tables des matières des manuels de FLE, articulent tant bien que mal les types de progression fonctionnelle et grammaticale, y mêlant également du notionnel et de l'actionnel, et il serait intéressant de reposer la question de cette articulation.

En l'absence de réponses définitives à ces questions, nous avons interrogé les méthodes appliquées par les enseignants, consciente qu'il existe probablement un décalage entre les pratiques de classe déclarées et les pratiques effectives.

\section{L'enquête}

49 enseignants de FLE ont répondu à notre sondage en mars 2017, tous enseignants belges francophones natifs enseignant le français langue étrangère.

Cette enquête interroge les pratiques déclarées des enseignants de FLE sur l'enseignement grammatical. Nous mettrons en parallèle nos résultats avec ceux de 
l'enquête de Fougerouse (2001), afin d'établir les convergences et divergences entre les deux sondages.

Figure 1 - Réponses à la question 1

\section{Vous enseignez le français langue étrangère}

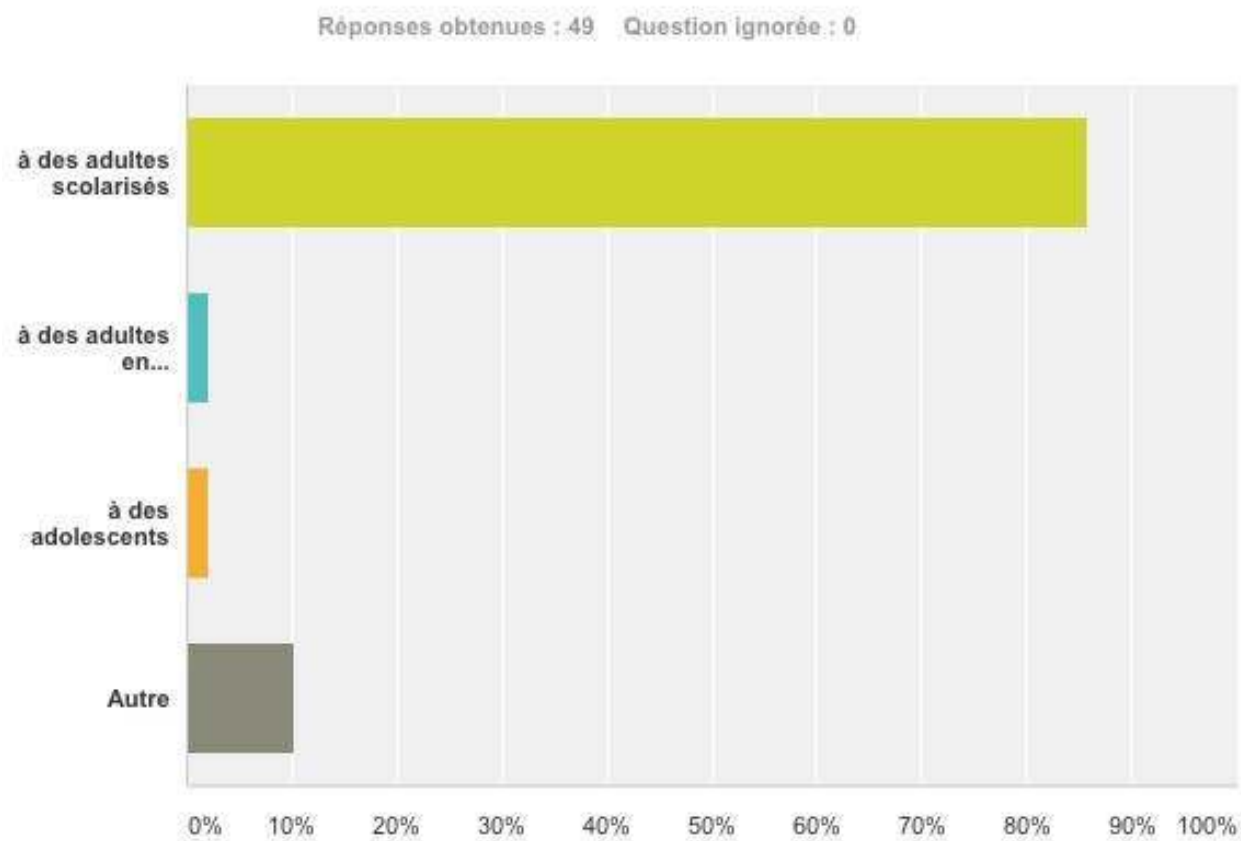

Plus des trois-quarts d'entre eux enseignent à des adultes scolarisés. Les autres enseignent à des adultes en alphabétisation, ou les deux (autre), à des adolescents, à des adultes en recherche de travail (autre) ou suivant des cours de promotion sociale (autre). 
Figure 2 - Réponses à la question 2

Vous enseignez le FLE à quel niveau ? (plusieurs réponses possibles)

Rèponsos obtenues : 49 Question ignorée: :

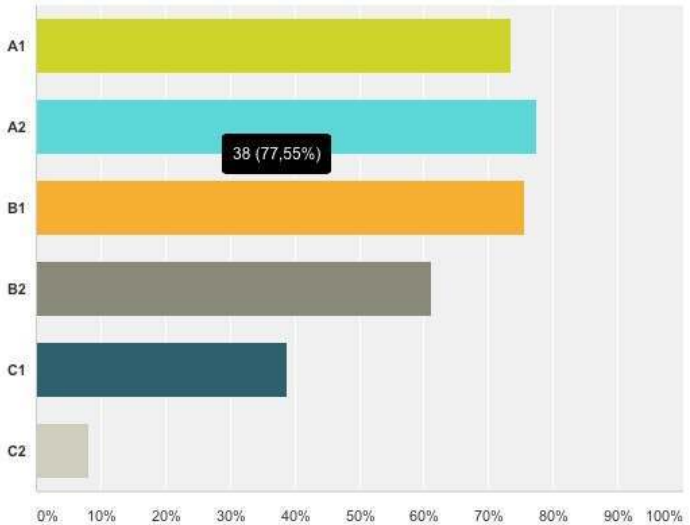

\begin{tabular}{|l|lc}
\hline Choix de réponses & Réponses & \\
\hline A1 & $\mathbf{7 3 , 4 7 \%}$ & 36 \\
\hline $\mathbf{A} 2$ & $\mathbf{7 7 , 5 5 \%}$ & 38 \\
\hline $\mathbf{B} 1$ & $\mathbf{7 5 , 5 1 \%}$ & 37 \\
\hline $\mathbf{B} 2$ & $\mathbf{6 1 , 2 2 \%}$ & 30 \\
\hline C1 & $\mathbf{3 8 , 7 8 \%}$ & 19 \\
\hline C2 & $\mathbf{8 , 1 6 \%}$ & 4 \\
\hline Nombre total de participants : 49 & & \\
\hline
\end{tabular}

29 Ces professeurs s'adressent essentiellement à des apprenants de niveaux de A1 à B2, souvent à plusieurs niveaux.

Figure 3 - Réponses à la question 3

\section{Consacrez-vous du temps aux explications de grammaire en classe?}

Réponses obtonues : 49 Question ignorèe : 0

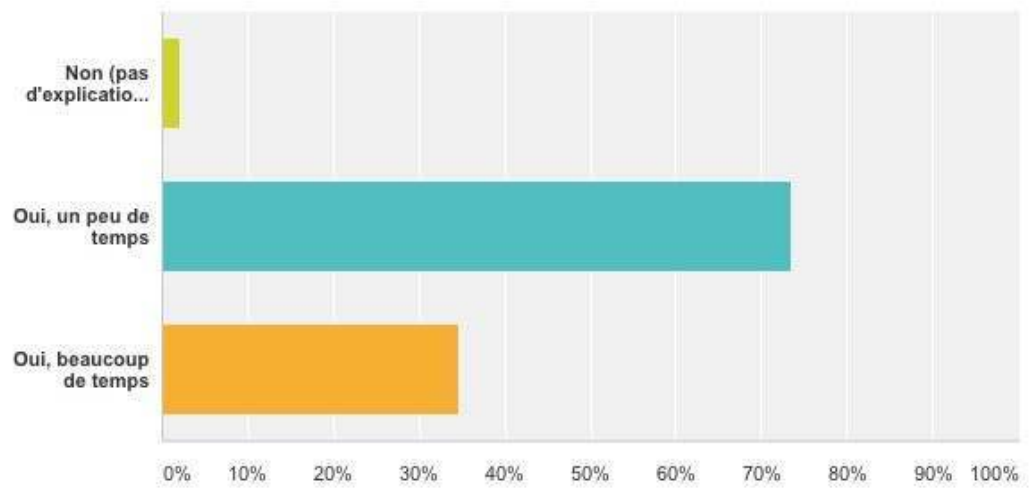

\begin{tabular}{|c|c|c|c|}
\hline Choix de réponses & $\mathrm{*}$ & Réponses & r \\
\hline Non (pas d'explication de grammaire) & & $2,04 \%$ & 1 \\
\hline Ouî, un peu de temps & & $73,47 \%$ & 36 \\
\hline Oui, beaucoup de temps & & $34,69 \%$ & 17 \\
\hline
\end{tabular}


Les trois-quarts des enseignants déclarent consacrer un peu de temps aux explications de grammaire, le dernier quart déclare y passer beaucoup de temps, et seul l'un d'entre eux déclare ne pas y consacrer de temps. Ces résultats signifient qu'il n'y a dans aucune des classes une méthodologie d'implicitation de la grammaire, nous voyons la confirmation ici que la période du silence métalinguistique, si tant est qu'elle ait réellement existé, est révolue, mais je pense que personne n'en doutait.

Les résultats de cette enquête divergent de ceux établis par Fougerouse (2001). L'enseignement semble proportionnellement moins grammatical : 30 à $50 \%$ du temps de classe était consacré à la grammaire, alors que les trois quarts des enseignants que nous avons sondés disent consacrer un peu de temps à la grammaire. Cette formulation est exprimée subjectivement afin de mettre en lumière les perceptions des enseignants et non une mesure objective du temps consacré à la grammaire, mesure qu'ils ne sont peutêtre pas à même de fournir avec certitude.

Figure 4 - Réponses à la question 4

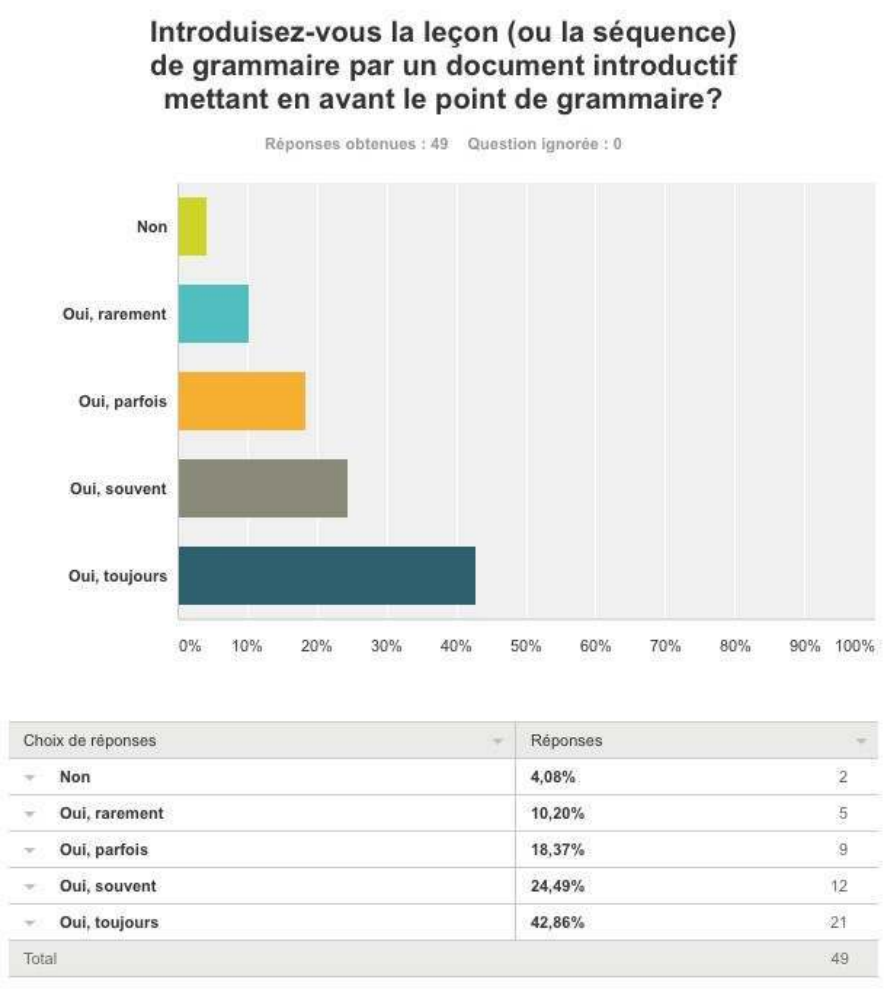

Ce schéma montre que les enseignants introduisent la leçon par le biais d'un document mettant en évidence le point de grammaire : plus de $40 \%$ le font toujours, $24 \%$ souvent, $18 \%$ parfois, $10 \%$ rarement et seuls deux enseignants ne le font pas. Un de ces deux enseignants a cependant répondu à la question suivante, qui impliquait pourtant de pratiquer l'activité décrite dans la question précédente. Nous en déduisons, avec Fougerouse, que :

pour eux (NDA : les enseignants), le sens prime sur l'aspect formel : une forme est d'abord abordée en contexte, avant d'être isolée puis analysée. Cette attitude révèle que dans les années 90 , les enseignants ne se sont pas contentés de réintroduire de la grammaire sous une forme traditionnelle dans la classe; ils ont également compris et intégré l'importance du sens dans la communication. (Fougerouse, 2001 : 169) 
33 À la question du type d'enseignement grammatical, environ un enseignant sur six répond qu'il fait toujours deviner la règle, environ un sur trois souvent et environ un sur trois parfois. L'enquête ne permet pas de savoir si ces enseignants pratiquent la conceptualisation ou non, mais nous pouvons affirmer que les autres font de l'induction, parfois ou souvent.

Cette façon de procéder a donc toujours cours aujourd'hui, même si elle est reconnue comme peu imaginable avec des apprenants totalement débutants. Elle implique en effet une intériorisation minimale du système de la langue-cible, ce qui a été précisé par un enseignant: comment peut-on discuter de règles sans connaître du tout la langue à apprendre? Il faut pouvoir compter sur des connaissances de base, un niveau A2 minimum.

Elle implique également une habitude scolaire d'explicitation des contenus grammaticaux. Sans cette dimension socioculturelle, la démarche inductive amoindrit ses chances d'aboutir aux résultats escomptés.

Figure 5 - Réponses à la question 5

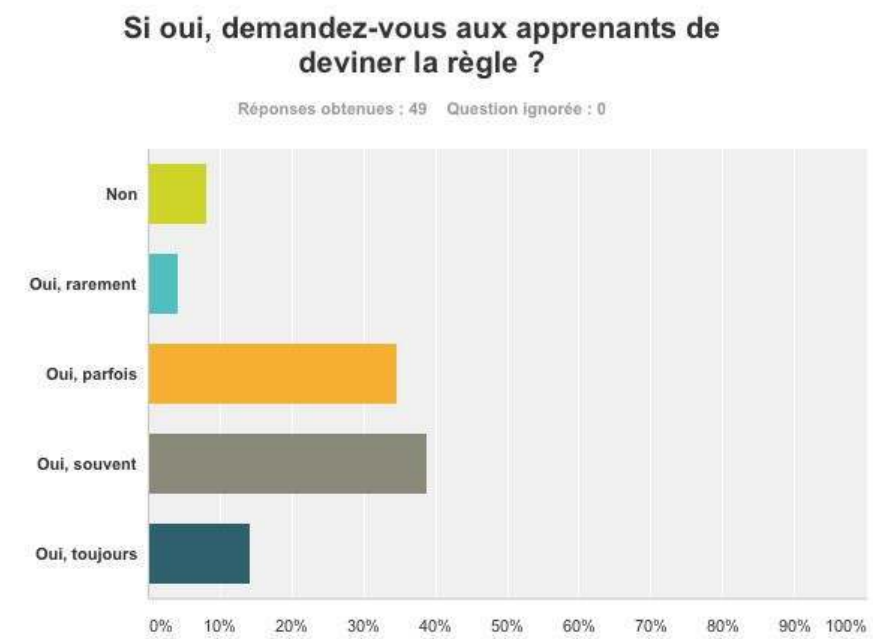

\begin{tabular}{|l|lc}
\hline Choix de réponses & Réponses & \\
\hline Non & $\mathbf{8 , 1 6 \%}$ & 4 \\
\hline Oui, rarement & $\mathbf{4 , 0 8 \%}$ & 2 \\
\hline Oui, parfois & $34,69 \%$ & 17 \\
\hline Oui, souvent & $\mathbf{3 8 , 7 8 \%}$ & 19 \\
\hline Oui, toujours & $\mathbf{1 4 , 2 9 \%}$ & 7 \\
\hline Total & & 49 \\
\hline
\end{tabular}

On peut affirmer que les activités relèvent de la conceptualisation grammaticale et non de la conceptualisation, puisque les enseignants reformulent, ré-expliquent, et/ou complètent la règle : plus de la moitié le fait toujours et seuls six enseignants ne le font jamais ou rarement.

37 Les résultats de cette enquête divergent ici de ceux établis par Fougerouse (2001). Elle constatait également que deux tiers des enseignants préfèrent la démarche déductive à l'inductive, alors que nous remarquons que l'induction est largement dominante dans nos résultats. 
Comme il s'agit ici de pratiques déclarées, il existe peut-être un décalage avec les pratiques effectives : les enseignants pourraient se conformer à l'idée qu'ils se font de ce que devrait être le type de démarche grammaticale à adopter. Il s'agit peut-être également d'une évolution dans les pratiques des enseignants.

Figure 6 - Réponses à la question 6

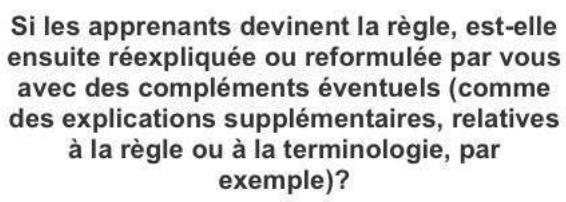

Si les apprenants devinent la règle, est-elle ensuite réexpliquée ou reformulée par vous avec des compléments éventuels (comme des explications supplémentaires, relatives à la règle ou à la terminologie, par exemple)?
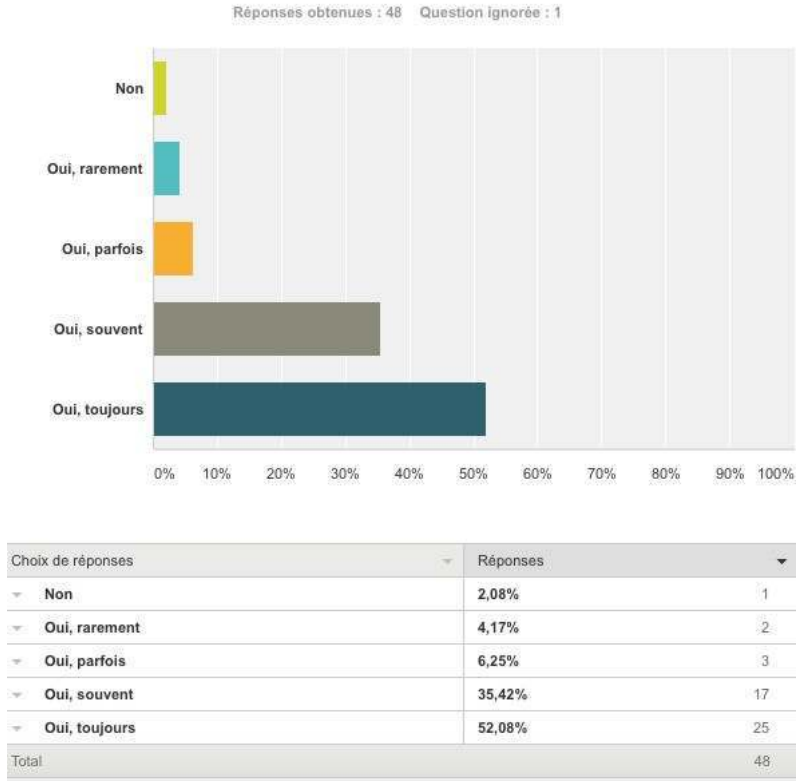

39 Nous avons demandé aux enseignants quels types d'activité relative à la grammaire ils pratiquent, passée la phase d'exposition de la règle. 
Figure 7 - Réponses à la question 7

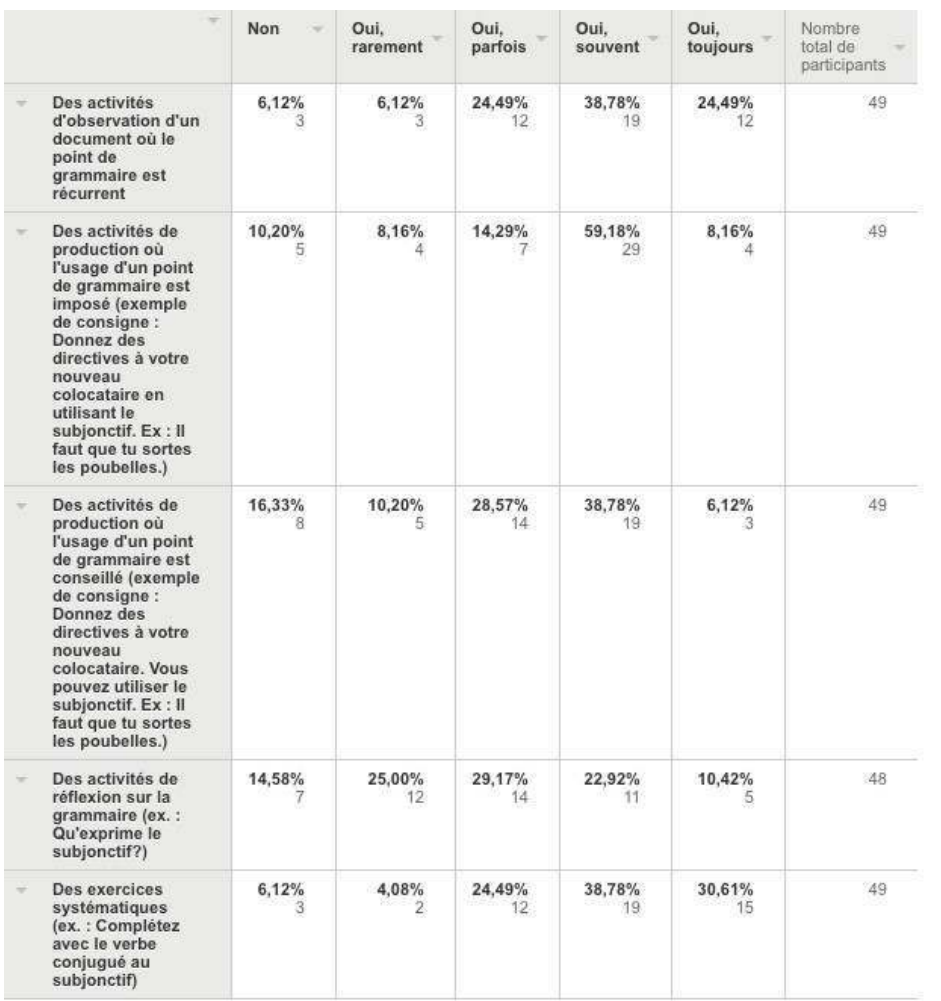

Ce schéma met en évidence la distribution des types d'activité : observation, production, réflexion, exercices systématiques. Les activités les plus largement partagées par les enseignants interrogés concernent la production langagière avec l'usage imposé d'un fait de langue et les exercices systématiques. Si ces deux types d'activités poursuivent l'objectif d'intériorisation du point de grammaire, les activités de production permettent la contextualisation communicative d'un point de grammaire alors que les exercices systématiques ne le permettent pas. Même s'ils sont issus des conceptions béhavioristes de l'apprentissage, qui considéraient ce type d'activité comme suffisant à l'acquisition, ils ne sont plus envisagés aujourd'hui qu'en complémentarité avec la démarche d'exposition à la règle et la contextualisation grammaticale :

La situation de communication fait partie intégrante du processus d'apprentissage de la grammaire. Les enseignants reconnaissent le lien étroit entre les deux : la situation de communication sert à introduire le point puis à le réinvestir (Fougerouse, $2001:$ 169-170).

41 Si l'induction est une pratique grammaticale explicite qui assume le fait de «faire de la grammaire » et d'y consacrer du temps, ce qui est peut-être plus facile dans le contexte actuel que dans celui de la fin du XX $\mathrm{XX}^{\mathrm{e}}$ siècle, marqué par les tentatives d'éviction de la grammaire. En témoignent les activités grammaticales au sens large, qui figurent aujourd'hui en bonne place dans les manuels. Plus d'induction aujourd'hui que dans l'enquête de Fougerouse, mais moins de conceptualisation.

L'activité la moins fréquemment mise en place est en effet la réflexion grammaticale. Comment interpréter ce résultat? Un manque d'intérêt des enseignants et/ou des apprenants, une absence de cohérence entre cet objectif et un objectif de développement de la compétence de communication, un manque de formation des enseignants, des apprenants ? Quoi qu'il en soit, cela illustre le fait que la conceptualisation grammaticale 
n'est pas présente dans les activités des enseignants sondés, contrairement à la démarche inductive. À cet égard, les résultats de Fougerouse sont très différents :

(...) les exercices de conceptualisation grammaticale sont entrés dans la classe de langue. Les deux tiers des enseignants disent les pratiquer, même si ce n'est pas systématiquement. (...) Cette manière de procéder présente une alternative à l'emploi du métalangage traditionnel, ce dernier n'étant pas absolument transparent et universel. (Fougerouse, $2001: 170$ )

Comment expliquer cette divergence méthodologique? D’autres activités, notamment dans une perspective d'enseignement communicatif par les tâches, ont peut-être évincé certaines activités de réflexion grammaticale. Ces tâches sembleraient peut-être moins métalinguistiques (à visée réflexive) que linguistiques (à visée de communication). Au lieu de mettre en place des activités de réflexion sur les langues, les enseignants consacreraient du temps à des activités de communication. Cette hypothèse cadrerait avec le fait que les enseignants dédieraient aujourd'hui moins de temps à la grammaire en général (voir plus haut).

\section{Pistes didactiques}

44 L'absence de données acquisitionnelles stables limite la dimension praxéologique de la didactique de la grammaire et on reste dans le doute face à la méthodologie à adopter en priorité, pour son efficacité objective. Il nous est cependant permis de repenser la question, finalement souvent absente, du discours grammatical en tant que tel, même si cela sort un peu du cadre de l'enquête proposée ici. Pour remplacer la grammaire traditionnelle, nous avons proposé un outil en ligne pour les étudiants allophones de l'Université libre de Bruxelles (voir Damar, Roig \& Van Raemdonck, 2010b) qui proposait des fiches de grammaire et des exercices. Cet outil n'envisageait pas de méthodologie particulière : il pouvait être investi en autoformation ou en classe avec une démarche inductive ou déductive. Les bénéfices cognitifs sont potentiellement immenses (absence de dissonance cognitive quand des énoncés pourtant corrects ne cadrent pas avec la règle intériorisée; cohérence interne du système; acquisition plus en adéquation avec le système linguistique de la langue cible; etc.), mais il faut s'interroger sur les résistances que ces pratiques ne manquent pas de rencontrer auprès des enseignants.

La relégation du - relatif - consensus actuel sur la terminologie grammaticale entraverait la progression des curriculums. Un apprenant pourrait éprouver des difficultés à passer d'une vision du système linguistique à une autre, difficultés inversement proportionnelles à sa familiarisation avec la formalisation grammaticale. On confond tellement souvent, et à tort, langue et description de la langue. Et ce serait faire peu de cas du temps, qui risque bien d'être à perte, à passer d'un discours à un autre, en fonction des enseignants et de leur vision du système linguistique. Le consensus, même sur une grammaire douteuse, risquerait d'être préféré à l'éclatement des cadres théoriques, qui nécessiterait des activités de réflexion grammaticale en amont.

Mais si l'on adoptait une attitude plus novatrice, les activités d'induction et de conceptualisation permettraient la révision du métalangage, et il pourrait être réduit au minimum, ce qui est souhaitable pour éviter de charger la barque grammaticale de l'apprenant : l'enseignant peut donner à voir des corpus montrant une vision du système moins faussée que celle offerte par la grammaire traditionnelle. Pour reprendre l'exemple, abordé plus haut, de l'enseignement du futur, l'enseignant montrera des 
exemples où les futurs sont proches ou lointains, en précisant que l'emploi du futur périphrastique (anciennement " proche ») engendre un effet qui est dû à sa forme même : la composition à partir du verbe « aller » conjugué au présent de l'indicatif. Ce temps (ou tiroir) grammatical étant celui prototypiquement associé à la réalité extralinguistique présente, l'effet produit par la forme est celui d'un engagement plus grand par rapport à la forme du futur simple, qui apparait comme plus détachée du présent. Il n'est nul besoin d'un métalangage lourd ni même de terminologie particulière pour faire saisir ce concept. Nous avons développé cette idée (Damar, à paraître) dans le cadre des activités du groupe de recherche Grammaire et Contextualisation (GRAC).

Finalement, le fait que les enseignants déclarent préférer l'induction nous laisse à penser que l'innovation grammaticale pourrait passer par des activités inductives et de conceptualisation, en donnant à voir les éléments d'une description revisitée du système linguistique. Cette démarche semble constituer une option susceptible de répercussions bénéfiques sur le système intériorisé. Il faudrait pour cela étudier les représentations des enseignants à propos du système grammatical et voir s'il est possible et, si oui, de quelle manière, on peut les faire évoluer. Cette option entrainerait probablement d'autres évolutions, plus globales, sur les représentations des méthodologies d'enseignement de la grammaire, sur les démarches inductives et de conceptualisation.

\section{BIBLIOGRAPHIE}

Berthoud, A.-C. \& Py, B. (1993). Des linguistes et des enseignants. Bern : Peter Lang.

Besse, H. (1986). « Enseignement-apprentissage des langues étrangères et connaissances grammaticales et linguistiques ». Les Langues modernes, $\mathrm{n}^{\circ} 2$. pp. 19-34.

Besse, H. \& Porquier, R. (1991, 1e éd.1984). Grammaire et didactique des langues. Paris : Didier-Hatier. Berthet, A., Hugot, C., Sampsonis, B., Waendendries, M. \& Kizirian, V.-M. (2006). Alter Ego 1. Paris : Hachette.

Coste, D. (1985). « Sur quelques aspects des relations récentes entre grammaire et didactique du FLE ». Langue française, $\mathrm{n}^{\circ}$ 68. pp. 5-16.

Cuq, J.-P. (1996). Une introduction à la didactique de la grammaire en FLE. Paris : Didier.

Damar, M.-E. (à paraître). « La grammaire du FLE ou la perte du sens grammatical : l'exemple des futurs simple et proche ", Acte du troisième colloque international du GRAC : "Enseigner la grammaire : discours, descriptions et pratiques ", 18-19 juin 2015 - Université d'Algarve, Faro (Portugal).

Damar, M.-E. (2008). Pour une linguistique applicable : l'exemple du subjonctif en FLE. Bruxelles : Peter Lang.

Damar, M.-E., Roig, A., \& Van Raemdonck, D. (2010a). « Faire l'article en FLE ». Anale Universit

Stefan cel Mare. Seria Filologie. A. Lingvistic, 2010/2, Tome XVI, n 1. pp. 79-90. 
Damar, M.-E., Roig, A. \& Van Raemdonck, D. (2010b). Outil d'auto-formation à la grammaire française en ligne pour étudiants allophones. VIIe Colloque des Technologies de l'Information et de la Communication pour l'Enseignement. URL : http://difusion.ulb.ac.be/vufind/Record/ULB, consulté le 30/10/2017.

Denyer, M., Garmendia, A., Royer, C. \& Lions-Oliviéri, M.-L. (2010). Version Originale A2. Paris : Maison des Langues.

De Keyser, R. (1995). "Learning second language grammar rules: an experiment with a miniature linguistic system". Studies in Second Language Acquisition, vol. 17, n 3. pp. 379- 410.

Ellis, R. (1993). "Second language acquisition research: how does it help teachers? An interview with Rod Ellis". ELT Journal, n 47/1. pp. 3-11.

Flament-Boistrancourt, D. (dir.). (1994). Théories, données et pratiques en FLE. Lille : Diffusion Presses Universitaires de Lille.

Fourgerouse, M.-C. (2001). « L'enseignement de la grammaire en classe de français langue étrangère ». Études de Linguistique Appliquée, n 122. pp. 165-179.

Houdebine-Gravaud, A.-M. (dir.). (2002). L'Imaginaire Linguistique. Paris : l'Harmattan.

Kasazian, E. (2017). Pratiques d'enseignement et descriptions grammaticales des langues étrangères dans le contexte scolaire : le cas de l'Angleterre. Thèse de doctorat non publiée. Paris : Université Paris III Sorbonne Nouvelle.

Germain, C. (1993). Évolution de l'enseignement des langues : 5000 ans d'histoire. Paris : Clé International.

Jodelet, D. «Représentations sociales : phénomènes, concepts et théories » in Moscovici, S. (1984). Psychologie sociale. Paris : PUF. pp. 363-384.

Ladmiral, J.-R. (1975). « Linguistique et pédagogie des langues étrangères ». Langage, $\mathrm{n}^{\circ} 39$. pp. 5-18.

Moscovici, S. (1984). Psychologie sociale. Paris : PUF.

Seliger, H. (1975). "Inductive method and deductive method in language teaching: a reexamination". International Review of Applied Linguistics, $\mathrm{n}^{\circ}$ 13. pp. 1-18.

Sharwood Smith, M. (1981). "Consciousness-raising and the second language learner". Applied Linguistics, vol. 11, $\mathrm{n}^{\circ} 2$. pp. 159-168.

Vigner, G. (1984). L'exercice dans la classe de français. Paris : Hachette.

Vigner, G. (2004). La grammaire en FLE. Paris : Hachette.

\section{ANNEXES}

\section{Questionnaire destiné aux enseignants}

\section{7 questions sur la grammaire dans vos classes de FLE : Une enquête auprès des enseignants}

1. Vous enseignez le français langue étrangère :

. à des adultes scolarisés

. à des adultes en alphabétisation 
. à des adolescents

. Autre

2. Vous enseignez le FLE à quel niveau? (Plusieurs réponses possibles)

. A1

. A2

. B1

. B2

. $\mathrm{C} 1$

. $\mathrm{C} 2$

3. Consacrez-vous du temps aux explications de grammaire en classe?

. Non (pas d'explication de grammaire)

. Oui, un peu de temps

. Oui, beaucoup de temps

4. Introduisez-vous la leçon (ou la séquence) de grammaire par un document introductif mettant en avant le point de grammaire?

. Non

. Oui, rarement

. Oui, parfois

. Oui, souvent

. Oui, toujours

5. Si oui, demandez-vous aux apprenants de deviner la règle?

. Non

. Oui, rarement

. Oui, parfois

. Oui, souvent

. Oui, toujours

6. Si les apprenants devinent la règle, est-elle ensuite réexpliquée ou reformulée par vous avec des compléments éventuels (comme des explications supplémentaires, relatives à la règle ou à la terminologie, par exemple)?

. Non

. Oui, rarement

. Oui, parfois

. Oui, souvent

. Oui, toujours

7. Parmi ces activités, lesquelles faites-vous?

Options de réponse par activité: Non / Oui, rarement / Oui, parfois / Oui, souvent / Oui, toujours . Des activités d'observation d'un document où le point de grammaire est récurrent . Des activités de production où l'usage d'un point de grammaire est imposé (exemple de consigne : Donnez des directives à votre nouveau colocataire en utilisant le subjonctif. Ex : Il faut que tu sortes les poubelles.)

. Des activités de production où l'usage d'un point de grammaire est conseillé (exemple de consigne : Donnez des directives à votre nouveau colocataire. Vous pouvez utiliser le subjonctif. Ex : Il faut que tu sortes les poubelles.)

. Des activités de réflexion sur la grammaire (ex. : Qu'exprime le subjonctif?) 
. Des exercices systématiques (ex. : Complétez avec le verbe conjugué au subjonctif) . Autre :

\section{RÉSUMÉS}

Après un éclairage théorique sur la problématique et les enjeux de l'enseignement grammatical, cette contribution entend, par le biais d'une enquête auprès d'enseignants de FLE belges francophones, apporter un éclairage sur les pratiques déclarées des enseignants sur les démarches d'enseignement de la grammaire : déductive, inductive ou par la conceptualisation. Si l'induction à partir d'un corpus semble constituer une pratique généralisée, ce type de méthodologie occulte la problématique de la pertinence du discours grammatical sous-jacent, qui oriente pourtant l'élaboration du corpus et le guidage des apprenants dans leurs hypothèses.

After a theoretical insight into the issues and challenges of grammar teaching, this contribution aims to shed light on the teachers' reported practices regarding approaches for teaching grammar, through a survey of teachers of FLE (French as foreign language) French-speaking Belgian. While induction from a corpus seems to be a widespread practice, this type of methodology conceals the problem of the relevance of the underlying grammatical discourse, which nevertheless guides the elaboration of the corpus and the guidance of learners in their hypotheses.

\section{INDEX}

Mots-clés : Enseignement, FLE, grammaire, induction, déduction

Keywords: Teaching, FFL, grammar, inductive instruction, deductive instruction

\section{AUTEUR}

\section{MARIE-EVE DAMAR}

Université libre de Bruxelles, Belgique

Marie-Eve Damar est docteure en Langues et Lettres, spécialisée dans l'enseignementapprentissage de la grammaire. Elle enseigne à l'Université libre de Bruxelles et à l'Université de Mons (Belgique) les disciplines suivantes : la linguistique, la linguistique appliquée et la communication écrite. Certaines de ses recherches interrogent les apports potentiels de la linguistique pour l'enseignement du FLE, et s'inscrivent dans le domaine novateur de la linguistique applicable.

Courriel : mdamar[a]ulb.ac.be 